\title{
¿Cómo ayuda la Pizarra Digital Interactiva (PDI) a la hora de promover prácticas de indagación y modelización en el aula de ciencias?
}

\author{
Víctor López ${ }^{1, a}$, Carme Grimalt-Álvaro ${ }^{1, b}$, Digna Couso ${ }^{1, c}$ \\ ${ }^{1}$ Centre per a l'Educació Científica i Matemàtica (CRECIM). Edifici GL-304. Facultat de Ciències de \\ l'Educació. Campus de la Universitat Autònoma de Barcelona. 08193 Bellaterra (Barcelona). España. \\ ${ }^{a}$ Victor.Lopez@uab.cat, ${ }^{b C a r m e . G r i m a l t @ u a b . c a t, ~ D i g n a . C o u s o @ u a b . c a t ~}$
}

[Recibido: 4 Diciembre 2017. Revisado: 19 Abril 2018. Aceptado: 2 Mayo 2018]

Resumen: La Pizarra Digital Interactiva (PDI), interfaz táctil conectada a un ordenador, permite no solo escribir o dibujar como en una pizarra tradicional sino también insertar y arrastrar imágenes, navegar o resaltar en pantalla. En este artículo analizamos el potencial de esta herramienta educativa para la enseñanza y aprendizaje de las ciencias en secundaria desde la perspectiva de la práctica científica, centrándonos en las esferas de indagación y modelización. Para ello, se han analizado 20 talleres experimentales de física, química y biología dirigidos a estudiantes de 13 a 17 años y que se llevan a cabo en un laboratorio informatizado. Estos talleres están estructurados en torno a un ciclo de aprendizaje que incluye las siguientes etapas: explorar los modelos iniciales del alumnado, diseñar experimentos, realizar predicciones e hipótesis, recoger y analizar datos experimentales y elaborar modelos consensuados en grupo. El análisis ha permitido caracterizar qué acciones con la PDI se utilizan en cada etapa del ciclo definido, e identificar aquellos episodios relevantes donde el potencial del dispositivo es clave a la hora de promover que los y las estudiantes se involucren en procesos de indagación y modelización.

Palabras clave: Pizarra Digital Interactiva; argumentación; indagación; modelización; educación secundaria; aplicaciones informáticas;

How does the Interactive Whiteboard (IWB) help in promoting practices of inquiry and modeling in science classroom?

\begin{abstract}
Interactive Whiteboards (IWB) are touch interfaces connected to a computer. As such, they enable the user not only to write and draw on the screen as with traditional whiteboards, but also to insert and drag pictures, as well as to navigate or highlight on the screen. In this paper, we analyze the potential of these educational tools in secondary school science education from the perspective of promoting students' participation in the scientific practices, focusing on inquiring and modelling. To this end, 20 physics, chemistry and biology experimental workshops have been analyzed. All these workshops were carried out in a computer-based laboratory and were addressed to 13 to 17 -year-old-students. The workshops are structured around a learning sequence that includes the following stages: exploration of students' previous ideas, design of experiments, development of predictions and hypothesis, experimental data gathering and analysis, and group elaboration of consensus models. The analysis has made possible to characterize which actions carried out with the IWB are used in each stage of the defined cycle, and to identify those relevant episodes where the potential of the device is key in encouraging students to participate in inquiry and modeling practices.
\end{abstract}

Keywords: Interactive Whiteboard; argumentation; inquiry; modelling; secondary education; computer applications

Para citar este artículo: López, V., Grimalt-Álvaro, C. y Couso, D. (2018) ¿Cómo ayuda la Pizarra Digital Interactiva (PDI) a la hora de promover prácticas de indagación y modelización en el aula de ciencias?. Revista Eureka sobre Enseñanza y Divulgación de las Ciencias 15(3), 3302. doi: 10.25267/Rev_Eureka_ensen_divulg_cienc.2018.v15.i3.3302

\section{Introducción y estado de la cuestión}

En el contexto escolar, el término Pizarra Digital Interactiva (PDI) hace referencia a una superficie interactiva que, conectada a un ordenador, permite mostrar información e interactuar con ella a través de un lápiz marcador o directamente con el dedo, según el dispositivo. Esta superficie interactiva funciona mediante un software específico y, a menudo,

\author{
Revista Eureka sobre Enseñanza y Divulgación de las Ciencias \\ Universidad de Cádir. APAC-Eureka. ISSN: 1697-011X \\ bttp:/ / dx.doi.org/10.25267/Rev_Eureka_ensen_divulg_cienc.2018.v15.i3.3302 \\ http:/ / reuredc.uca.es
}


incorpora un paquete de presentación de información con una apariencia similar a la de un programa de presentación de diapositivas. Este dispositivo permite escribir sobre su superficie igual que una pizarra tradicional y puede usarse para proyectar texto o imágenes como en un proyector no interactivo. A su vez, permite interactuar con los objetos que se muestran (arrastrar imágenes insertadas y trazos realizados) (Kennewell y Beauchamp 2007; Aflalo et al. 2017) y sobrescribir encima de la información mostrada (Miller et al. 2005). Además, se puede almacenar las producciones en forma de diapositivas y recuperarlas en cualquier momento, e incluso permite generar un pequeño vídeo en el que se muestre el proceso de generación de estas diapositivas (Beauchamp y Parkinson 2005).

La implantación de la PDI en las escuelas de nuestro entorno ha ido creciendo dadas las múltiples potencialidades que ofrece en el aula, a nivel de flexibilidad, interacción o visualización (Hennessy y London 2013). No obstante, los diversos resultados de la literatura muestran que, el hecho de disponer de una PDI en el aula no implica necesariamente la mejora del aprendizaje del alumnado, sino que depende completamente de la manera con la que esta herramienta se usa (Pedró 2011). Así, dada la diversidad de situaciones y visiones, es necesario considerar cuáles son las potencialidades más significativas de la PDI desde el marco de la práctica científica escolar, tal y como se expone a continuación.

\section{Indagar y modelizar en el aula de ciencias}

Desde la tradición más social del aprendizaje y el paradigma sociocultural, la enseñanza y aprendizaje de las ciencias se conciben como una práctica social, que requiere de la interacción entre individuos y la participación de éstos en una comunidad (Chamizo e Izquierdo 2005). Esta afirmación se argumenta tanto por el hecho que el aprendizaje ocurre en gran medida en el plano social y discursivo (Vygotsky 1978), como porque las propias ciencias son en sí mismas una práctica social y discursiva (Osborne 2014; Kelly 2013). Por lo tanto, las interacciones en el plano social que se dan en la clase de ciencias ofrecen al alumnado la oportunidad de desarrollar sus habilidades imitando las acciones que realizan otros compañeros y el profesorado, discutiendo las tareas que se llevan a cabo y haciendo visible el propio pensamiento en una comunidad (Roschelle et al. 2001).

Partiendo de este enfoque, actualmente numerosos autores abogan por una enseñanza y aprendizaje de las ciencias que implique no sólo consumir los productos derivados de la actividad científica profesional, sino participar de la práctica social, discursiva y cognitiva que lleva asociada la actividad científica (Duschl y Grandy 2012; Kelly 2013; Osborne 2014). Esta manera de entender las clases de ciencia se refleja en el nuevo currículum de los Estados Unidos de América, donde la National Research Council (2012) concreta ocho prácticas científicas a promover en el aula: plantearse preguntas sobre fenómenos naturales relevantes para la ciencia, desarrollar y usar modelos científicos, planificar y llevar a cabo investigaciones, analizar e interpretar datos experimentales, usar pensamiento computacional y matemático, construir explicaciones y diseñar soluciones, argumentar científicamente en base a pruebas y comunicar a la comunidad los resultados de la actividad científica. Así, las clases de ciencias no deben centrarse en la asimilación de contenidos que transmite el docente (las ideas o modelos científicos descritos en el libro de texto tradicional), sino en el proceso de construcción y refinamiento de las explicaciones o modelos que construyen progresivamente los estudiantes sobre los fenómenos del mundo natural a los que se enfrentan. Minner, Levy, y Century (2010) afirman que esta manera de hacer ciencias no solo puede ayudar al desarrollo del aprendizaje conceptual, sino que es necesario para el desarrollo de habilidades propias de la actividad científica.

En base a estos planteamientos, y coincidiendo con la idea de Izquierdo, Espinet, García, Pujol, y Sanmartí (1999) según la que el verdadero aprendizaje de la ciencia escolar es, en el 
fondo, el proceso de "pensar, hacer y hablar" ciencia, Osborne (2014) propone considerar tres esferas interrelacionadas de la práctica científica en el aula: modelización, indagación y argumentación. Así, la modelización puede entenderse como el proceso que implica desarrollar, evaluar y refinar explicaciones científicas sobre fenómenos naturales (Justi 2006; Gutiérrez 2004), la indagación es el proceso que conlleva diseñar y llevar a cabo experimentos y analizar e interpretar datos (Windschitl et al. 2008; Minner et al. 2010; Couso y Garrido 2016); y la argumentación puede entenderse como comunicar las ideas propias y las interpretaciones de los resultados para persuadir a los demás de su validez (Duschl y Osborne 2002; Erduran y Jiménez-Aleixandre 2007).

\section{Indagar y modelizar en el aula de ciencias con la PDI}

Si la PDI es realmente una herramienta mediadora de la actividad escolar (ya que promueve la interacción entre estudiantes, su participación e implicación en las discusiones, la representación de sus ideas mediante trazos, etc.), podemos pensar que se trata de una herramienta especialmente privilegiada para involucrar a los estudiantes en el desarrollo de prácticas científicas de modelización e indagación. Si bien esta cuestión no ha sido todavía analizada en profundidad, sí que existen algunos indicios que señalan este potencial. Por ejemplo, Murcia (2014) describe cómo los usos con la PDI de ocultar y mostrar información y mostrar visualizaciones interactivas, multimodales y atractivas, pueden promover oportunidades para explicitar el conocimiento previo del alumnado, la exploración de ideas científicas y la generación de explicaciones sobre fenómenos naturales. Del mismo modo, Higgins, Wall, y Smith (2005) subrayan que las capacidades gráficas de la PDI y, en particular, la visualización de conceptos abstractos, puede ayudar al alumnado a la construcción de ideas científicas. Además, Mercer, Hennessy y Warwick (2010) describen cómo la PDI, con la función de escribir, facilita la orquestación del diálogo y la construcción de ideas de manera colectiva, de forma coherente a lo que Mortimer y Scott (2003) definieron como el proceso dialógico de construcción de significados científicos en el aula. Esta interacción colaborativa y dialógica, según Hennessy y London (2013) puede facilitar la discusión en grupo y el establecimiento de un consenso sobre la interpretación de un fenómeno, por ejemplo, a través del uso de distintos colores en los trazos que los estudiantes realizan cuando salen a escribir a la pizarra.

Otro gran argumento que nos lleva a pensar que la PDI puede ser una interesante herramienta para involucrar a los estudiantes en prácticas de modelización e indagación es la posibilidad que ofrece esta herramienta para comunicar y mostrar información multimodal y representaciones semióticas diversas. Esta posibilidad que ofrece la PDI también ha sido señalada en la literatura del ámbito (Kung Teck 2013; Murcia 2014; Hennessy y London 2013) y es, de hecho, una de las potencialidades más reconocidas por el propio profesorado de ciencias cuando se le pregunta por sus beneficios educativos (Grimalt-Álvaro 2016). A nuestro entender, el hecho que la PDI permita representar una amplia variedad de modos de representación semiótica (lenguaje verbal, imágenes y lenguaje visual, diagramas, gráficos o fórmulas matemáticas) la hace todavía más interesante para promover la modelización e indagación en el aula a través de los lenguajes de la ciencia (Lemke 1990), así como para combinar y entrelazar los distintos niveles de representación propios del lenguaje científico propuestos por Johnstone (1991): macroscópico u observable, microscópico, submicroscópico o atómico y simbólico. De hecho, de acuerdo con Evagorou, Erduran y Mäntylä (2015), si pretendemos que el alumnado participe de la práctica social, discursiva y cognitiva que lleva asociada la actividad científica, la enseñanza de las ciencias no sólo debería involucrar el "consumo" pasivo de estas representaciones por parte del alumnado, sino involucrarlo en el proceso activo de producción y generación de representaciones visuales propias producto de la actividad científica que se desarrolla en el aula. ¿Qué mejor herramienta que la PDI para 
facilitar la producción colaborativa de estas representaciones científicas?

Finalmente, la PDI como herramienta que ayuda a guiar el desarrollo de una secuencia de enseñanza - aprendizaje (Aflalo et al. 2017) puede también ser una herramienta interesante para planificar y elaborar procesos que requieren de una cierta secuencialidad, como lo son los procesos característicos de las prácticas indagativas: hacerse preguntas sobre fenómenos científicos relevantes, diseñar experimentos para investigarlos, recolectar datos, analizarlos y elaborar conclusiones a partir de ellos (Windschitl et al. 2008). En este sentido, Osborne y Hennessy (2003) destacan cómo la capacidad de la PDI para mostrar información de manera inmediata puede ayudar a la experimentación en el aula.

\section{El problema: la falta de concreción sobre el uso de la PDI para modelizar e indagar}

A pesar de haber encontrado múltiples indicios en la literatura disponible sobre el uso educativo de la PDI, echamos de menos una concreción sobre cómo usarla para involucrar a los estudiantes en prácticas de modelización e indagación. Es decir: echamos de menos una mirada a la Pizarra Digital propia del área de didáctica de las ciencias que ponga el énfasis en cómo la PDI realmente puede convertirse en una herramienta que ayude a involucrar a los estudiantes a "hacer" ciencia. De hecho, en la extensa revisión bibliográfica de Ormanci, Cepni, Deveci y Aydin (2015) sobre el uso de la PDI en las clases de ciencia también echamos en falta las perspectivas de modelización e indagación.

La ausencia de esta mirada propia de la didáctica de las ciencias hacia el uso de la PDI, en cierto modo, es coherente con el uso que actualmente se hace de esta herramienta en las aulas, que Hennessy y London (2013) denominan como "más bien pobre". Este hecho no es algo nuevo, ya que sin una mirada de cómo la PDI puede contribuir a la enseñanza y aprendizaje de las ciencias, el profesorado carece de guías sobre cómo utilizarla, y acaba adaptando su utilización a herramientas que ya conoce, como el proyector tradicional (Area-Moreira et al. 2016; López Simó et al. 2017). Esta falta de concreción contrasta, en cambio, con la extensa literatura existente en torno a cómo usar en el aula de ciencias otras tecnologías digitales como videojuegos, simulaciones, captadores de datos o dispositivos móviles (Crook, Sharma y Wilson 2015; Linn 2003; Pintó, Couso y Hernández 2010; Romero y Quesada 2014; Smetana y Bell 2011; Webb 2005). Además, creemos que aportar esta mirada propia al uso de la PDI desde la práctica de la modelización y la indagación, en el fondo, es una manera de ayudar a desarrollar el denominado Conocimiento Técnico Pedagógico del Contenido (Koehler y Mishra 2009).

\section{Pregunta de investigación}

Con el objetivo de desarrollar esta mirada a la PDI desde la perspectiva de la modelización y la indagación escolar, así como ofrecer al profesorado recursos y buenas prácticas que ayuden a aprovechar las diversas potencialidades de la PDI, nos planteamos la siguiente pregunta: ¿Cómo ayuda la Pizarra Digital Interactiva (PDI) a la hora de promover prácticas de indagación y modelización en el aula de ciencias?

\section{Metodología de la investigación}

\section{El contexto de estudio: el proyecto REVIR}

El proyecto REVIR (Realidad-Virtualidad) ofrece al alumnado y al profesorado de ESO y Bachillerato (12-17 años) talleres experimentales en la Universidad Autónoma de Barcelona de 3-4 horas, relacionadas con contenidos del currículum de física, química y biología. Con una trayectoria de más de 10 años, estos talleres se diseñan, se evalúan y se revisan de forma 
iterativa por parte de expertos en didáctica de las ciencias, con el objetivo de ofrecer propuestas educativas que promuevan la modelización y la indagación, así como una interacción altamente dialógica y una presentación del contenido a través de contextos relevantes para el estudiante. Además, su implementación se realiza en un laboratorio altamente digitalizado, con sensores para recoger datos, simulaciones virtuales, consolas táctiles y una PDI. Tanto este enfoque metodológico como este equipamiento informático suscitan un importante interés entre los centros próximos a la instalación, que se concreta con más de 2000 visitas de estudiantes cada año. Todos estos motivos hacen del proyecto REVIR un buen contexto para analizar el papel que juega la PDI en las tres esferas de la práctica científica anteriormente discutidas.

\section{Recogida y análisis de datos}

El presente estudio se basa en el trabajo iniciado en Bozzo, Grimalt-Álvaro y López (2015). Se grabaron en vídeo 20 talleres REVIR de diversos contenidos (física, química y biología) a lo largo del curso 2014-15, con una participación media de 25 estudiantes por taller guiados siempre por un docente (a lo largo de los 20 talleres intervinieron 2 hombres y 2 mujeres), usando una cámara enfocada a la PDI. Del total de 70 horas correspondientes a los 20 talleres se grabaron, 14,5 horas correspondientes a los momentos en los que el docente o los estudiantes utilizaban la PDI, ya que el resto del tiempo los estudiantes trabajaban en grupos pequeños en sus mesas. Estas 14,5 horas se dividieron en 340 clips independientes, que posteriormente fueron categorizados según dos dimensiones, construyendo así una matriz de análisis de clips $5 \times 7$, donde el código de cada clip corresponde a la combinación de una letra y un número (por ejemplo [1A]). El proceso de análisis fue realizado de forma independiente por dos investigadores, para garantizar un mayor nivel de validez del análisis. El sistema de categorización fue:

(I) Según la acción con la PDI observada:

[A] Proyectar información igual que un proyector tradicional

[B] Dibujar y escribir igual que en una pizarra tradicional

[C] Arrastrar y soltar objetos (imágenes y trazos)

[D] Sobrescribir y resaltar encima de la información proyectada

[E] Almacenar producciones y recuperarlas más adelante

(II) Según la etapa REVIR, codificando cada clip del 1 al 7.

[1] Presentar situación problema y tratar ideas previas del alumnado

[2] Planificar diseño experimental y recogida de datos

[3] Elaborar predicciones e hipótesis

[4] Visualizar y analizar los resultados experimentales

[5] Visualizar e interactuar con simulaciones virtuales

[6] Sintetizar, consensuar y elaborar conclusiones

[7] Usar conclusiones para responder a la situación problema inicial u otras de nuevas

Con los 340 clips codificados y distribuidos dentro de la matriz de análisis 5x7, se reconstruyeron las secuencias de uso de la PDI con las que se encadenaban los clips uno detrás de otro, identificando así 21 combinaciones diferentes de cadenas de clips, denominadas episodios. Estos 21 episodios se han representado en la Figura 1. Cada episodio puede darse o bien en un único taller o bien en varios talleres a la vez. Por ejemplo, el episodio [1B] -> [1C] 
$->[1 \mathrm{D}]->[1 \mathrm{C}]$ es muy común en la mayoría de talleres, y en algunos casos la cadena se alarga $\mathrm{a}[1 \mathrm{~B}]->[1 \mathrm{C}]->[1 \mathrm{D}]->[1 \mathrm{C}]->[1 \mathrm{D}]->[1 \mathrm{C}]$.

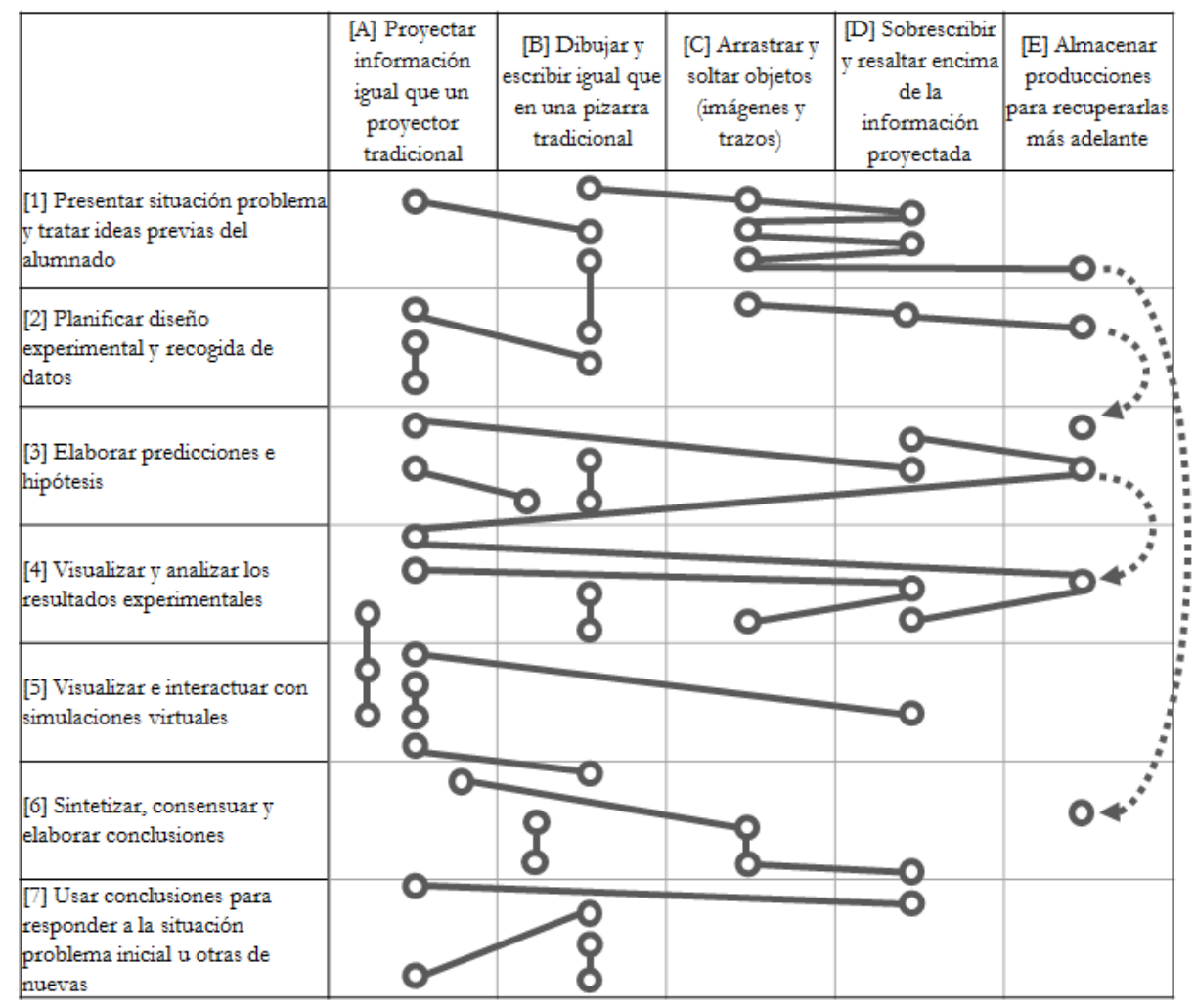

Figura 1. Representación de los 21 tipos de episodios a través de los cuales se encadenan los 340 clips en la matriz 5x7. Los círculos representan los momentos clave de cada episodio, incluyendo el inicio, el final y el momento en que se cambia de acción o de etapa REVIR. Las líneas continuas representan el encadenamiento temporal directo entre los momentos clave. Las líneas discontinuas de la derecha corresponden a los encadenamientos que no se dan uno inmediatamente después del otro, sino que al tratarse de la acción "almacenar reproducciones y recuperarla más adelante". Son los momentos en que el profesor recupera las producciones (dibujos, texto, gráficos, etc.) que el estudiante ha hecho un rato antes.

\section{Resultados}

En la Figura 1 aparece representada esta matriz de análisis 5x7 y cómo los 340 clips se encadenan a través de 21 tipos de episodios distintos, es decir, los 21 tipos de encadenamientos entre acciones (de la 1 a la 7 ) y etapas (de la A a la E). En esta figura puede observarse que la mayoría de episodios (13 de 21) sólo se sitúan en acciones tipo [A] y [B]. Es decir, aquellos que podrían haberse realizado de forma prácticamente igual si, en vez de PDI, el aula simplemente dispusiera de un proyector y una pizarra tradicional. Esto, en cierto modo, coincide con el análisis realizado por Aflalo, Zana y Huri (2017) en el que la mayoría de episodios observados con un grupo de profesores de ciencias usando la PDI corresponden a trazos sobre fondo blanco (lo que en nuestro caso es la acción tipo [B] o a proyección de vídeos e imágenes (lo que clasificamos como [A]), confiriendo una mayor validez a los resultados.

En la Figura 2 hemos destacado en colores los 5 episodios donde predominan las acciones tipo $[\mathrm{C}],[\mathrm{D}]$ y $[\mathrm{E}]$, ya que son acciones donde el aprovechamiento de la PDI es significativo (se realizan acciones que no serían posible sin este dispositivo). 


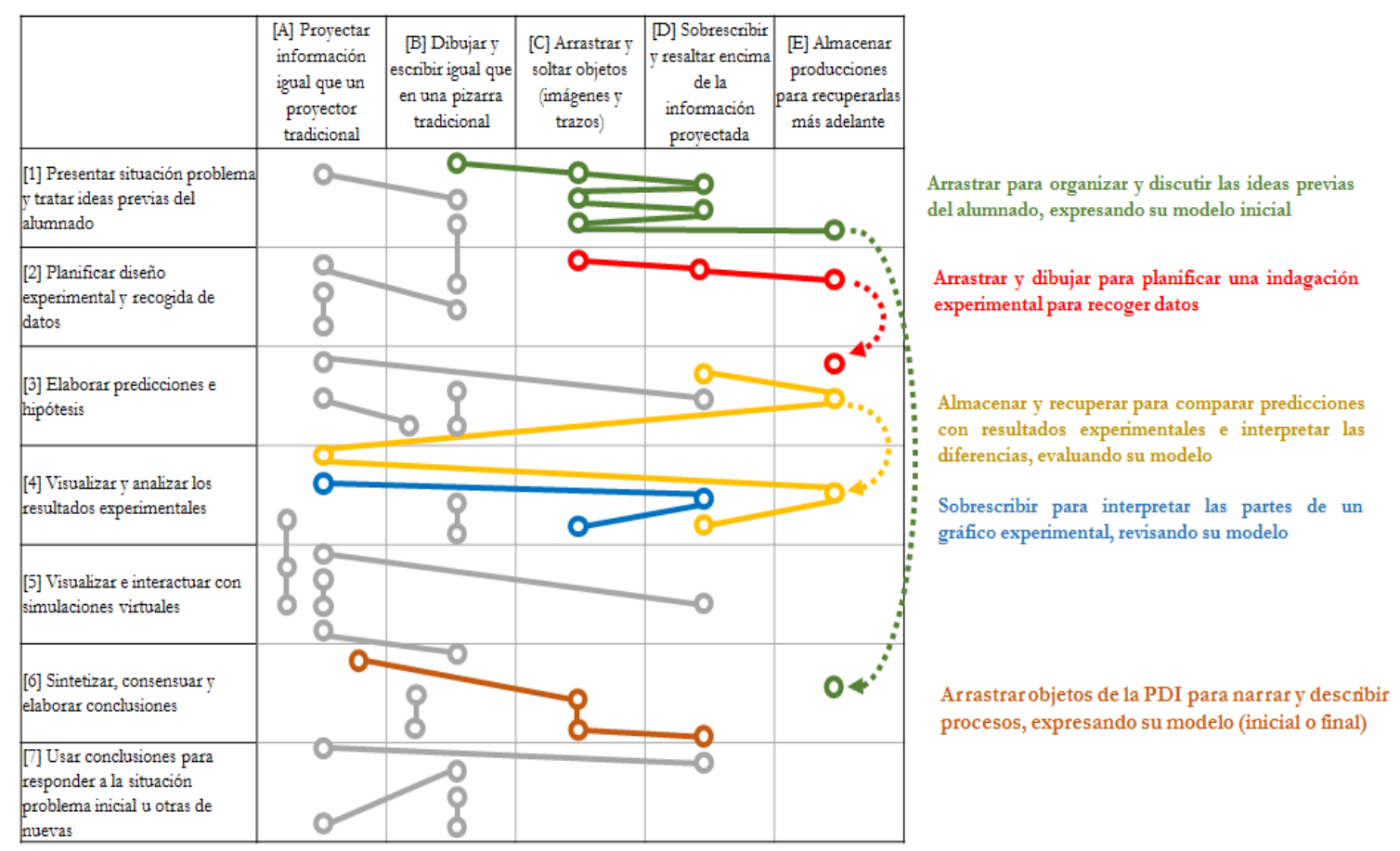

Figura 2. Se destaca en diversos colores la representación de los 5 episodios que incluyen acciones tipo [C], [D] y [E] y que se han seleccionado como relevantes para el presente estudio.

A continuación, se presentan y se discuten estos 5 episodios seleccionados. Para ello, se discute un ejemplo concreto correspondiente a uno de los talleres REVIR, si bien como hemos señalado anteriormente, el mismo episodio puede darse en más de un taller a la vez, con contenidos científicos distintos. Además, en cada episodio se muestran unas representaciones visuales que ilustran el episodio descrito (Figuras 3, 4, 5, 6 y 7). Estas representaciones son reproducciones hechas ad hoc para este artículo, que reproducen con fidelidad y calidad gráfica tanto las producciones escritas en la PDI como la situación docente y han sido traducidas del catalán al castellano.

\section{Arrastrar para organizar y discutir las ideas previas del alumnado, expresando su modelo inicial}

La Figura 3 recoge dos momentos distintos de la primera etapa de una sesión REVIR sobre termodinámica (energía, calor y trabajo). Al principio se pregunta al grupo que describan todas las formas imaginables de calentar una botella de agua (uso [1B]). A continuación, un segundo estudiante sale a la pizarra a clasificar las ideas de sus compañeros, arrastrando cada palabra a izquierda y derecha de la pantalla [1C] en función de la similitud entre las distintas formas de calentar la botella. Posteriormente, un tercer estudiante sale a la pizarra para escribir en un color distinto encima de la imagen resultante de la clasificación [1D] cual es el denominador común de todas las maneras de calentar agua. Esto permite construir en grupo más adelante [6E] la idea que las transferencias de energía pueden realizarse mediante «un cuerpo caliente» (listado de la izquierda) o mediante «un cuerpo haciendo fuerza» (listado de la derecha), clasificación de especial interés en la física para terminar conceptualizando el calor y el trabajo como los dos mecanismos de transferencia de energía. 


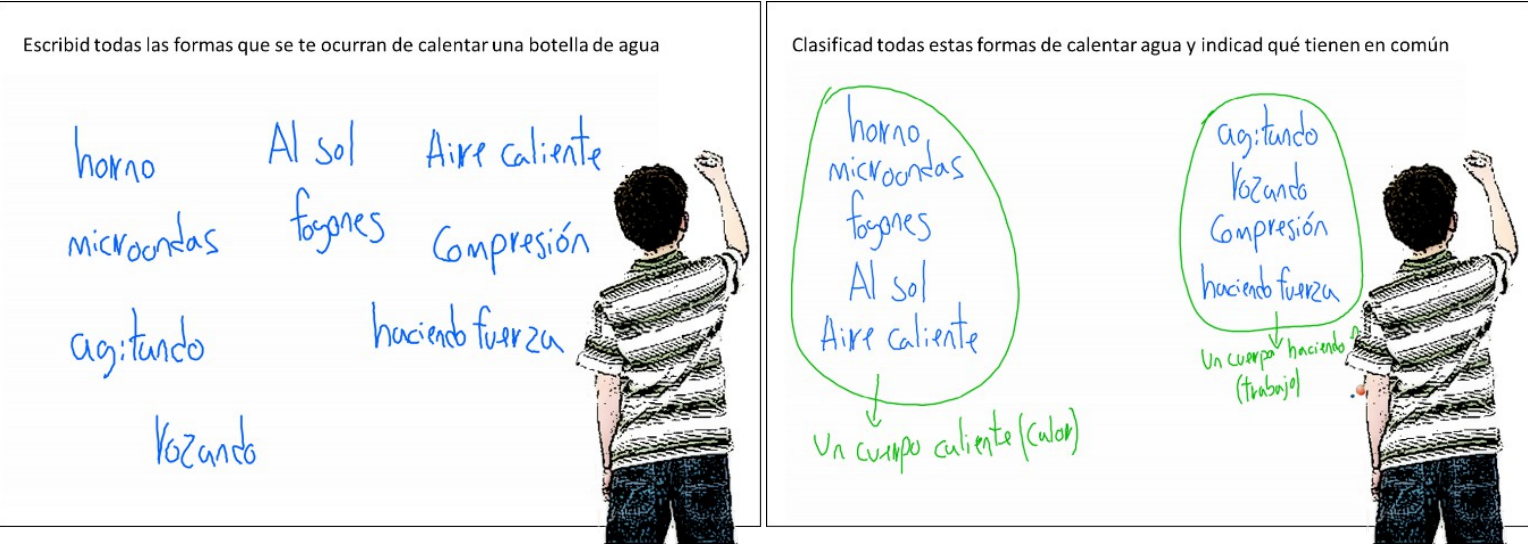

Figura 3. Izquierda: un primer estudiante anota un listado de formas de calentar una botella de agua. Derecha: otro estudiante organiza las formas según sus ideas sobre transferencia de energía.

\section{Arrastrar y dibujar para planificar una indagación experimental para recoger datos}

En este episodio se invita al alumnado a diseñar un montaje experimental para investigar los mecanismos de regulación térmica de los mamíferos (p. ej. la lana en las ovejas). Inicialmente se muestra una serie de elementos o piezas que se encuentran en el laboratorio, y se les propone que armen el sistema arrastrando las piezas sobre la pizarra [2C], escribiendo encima para aportar información sobre este montaje [2D]. Esto es justamente lo que se muestra en la Figura 4, donde los diferentes colores corresponden a las contribuciones hechas por otros dos estudiantes que salen a la pizarra para discutir cómo se controlan las variables y como se recogen y analizan los datos en su experimento. Esta producción la recuperarán más adelante $[3 \mathrm{E}]$ a la hora de predecir qué recipiente se enfriará antes.

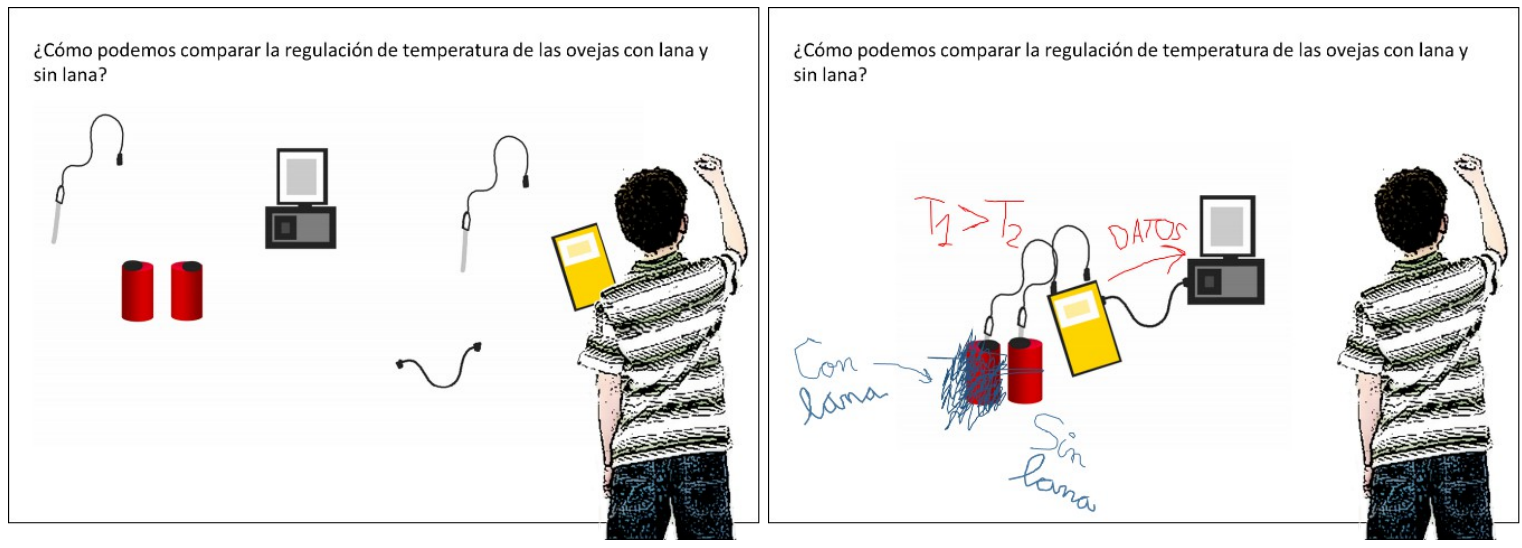

Figura 4. Izquierda: muestra una serie de elementos o piezas que se encuentran en el laboratorio para hacer un pequeño experimento. Derecha: varios estudiantes diseñan el experimento controlando variables y discutiendo la toma de datos experimentales.

\section{Almacenar y recuperar para comparar predicciones con resultados experimentales e interpretar las diferencias, evaluando su modelo}

En este episodio los estudiantes están investigando sobre el movimiento de un coche de juguete (cinemática). En un momento dado, 4 grupos de estudiantes salen a la pizarra a dibujar, sobre una plantilla con unos ejes de coordenadas ya preparados [3D], sus predicciones sobre cómo será el gráfico que obtendrán al medir experimentalmente el movimiento del cochecito con un sensor de movimiento. Estas predicciones quedan automáticamente almacenadas [3E] para poderse recuperar posteriormente. Así, después del experimento, el profesor muestra el gráfico experimental obtenido con un sensor de movimiento [4A], y a su vez recupera los dibujos realizados para compararlos con los resultados obtenidos [4E]. 
Finalmente, dos estudiantes salen de nuevo a la pizarra a sobre-escribir [4D] su análisis de los datos (similitudes y diferencias entre predicción y resultado).
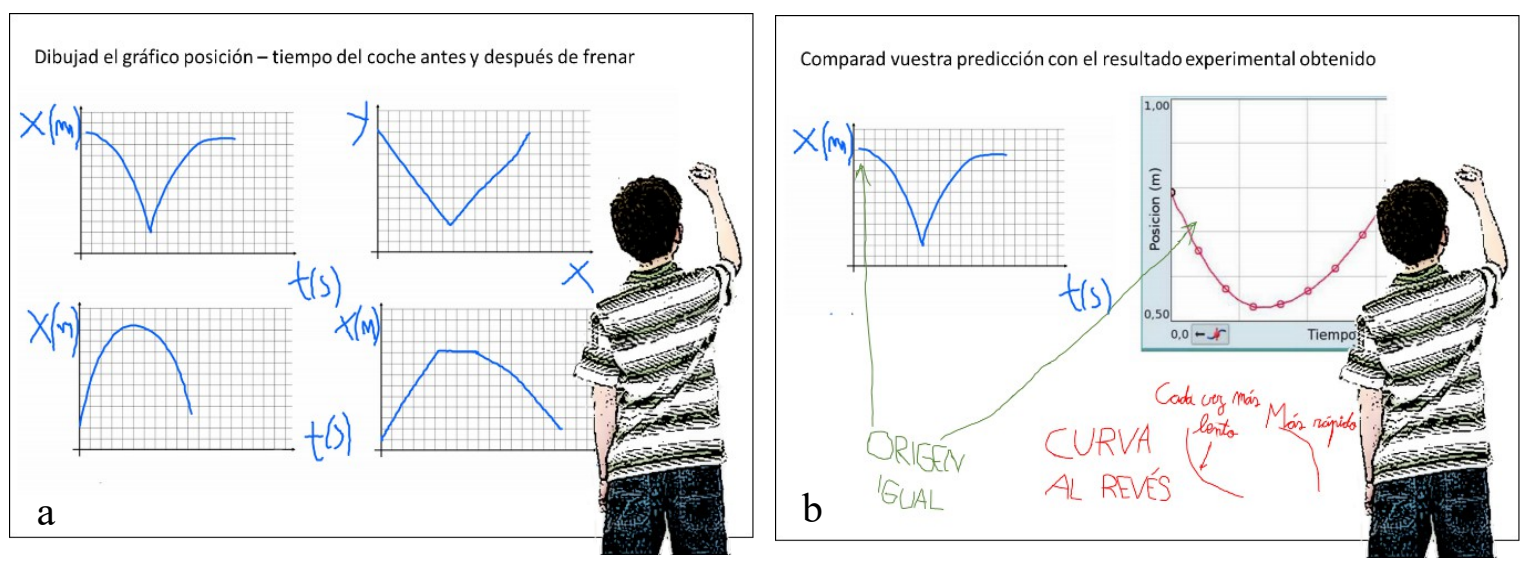

Figura 5. Izquierda: cuatro grupos de estudiantes anotan, sobre una plantilla, sus predicciones. Derecha: dos estudiantes comparan una de las predicciones con el resultado experimental obtenido.

\section{Sobrescribir para interpretar las partes de un gráfico experimental, revisando su modelo}

En este episodio primero se proyecta en la PDI el gráfico experimental obtenido por un grupo de alumnos, que en caso de la Figura 6a corresponde al gráfico experimental que se obtiene con el calentamiento del freno de una rueda de bicicleta mediante un software específico que permite conectar la PDI a un sensor de temperatura periférico [4A]. Posteriormente, tal como se muestra en la Figura 6b, se ve cómo este gráfico es analizado por estos mismos estudiantes, que sobrescriben sobre la imagen para identificar sus partes, anotar detalles, elaborar inferencias sobre su evolución en el tiempo, etc. [4D].

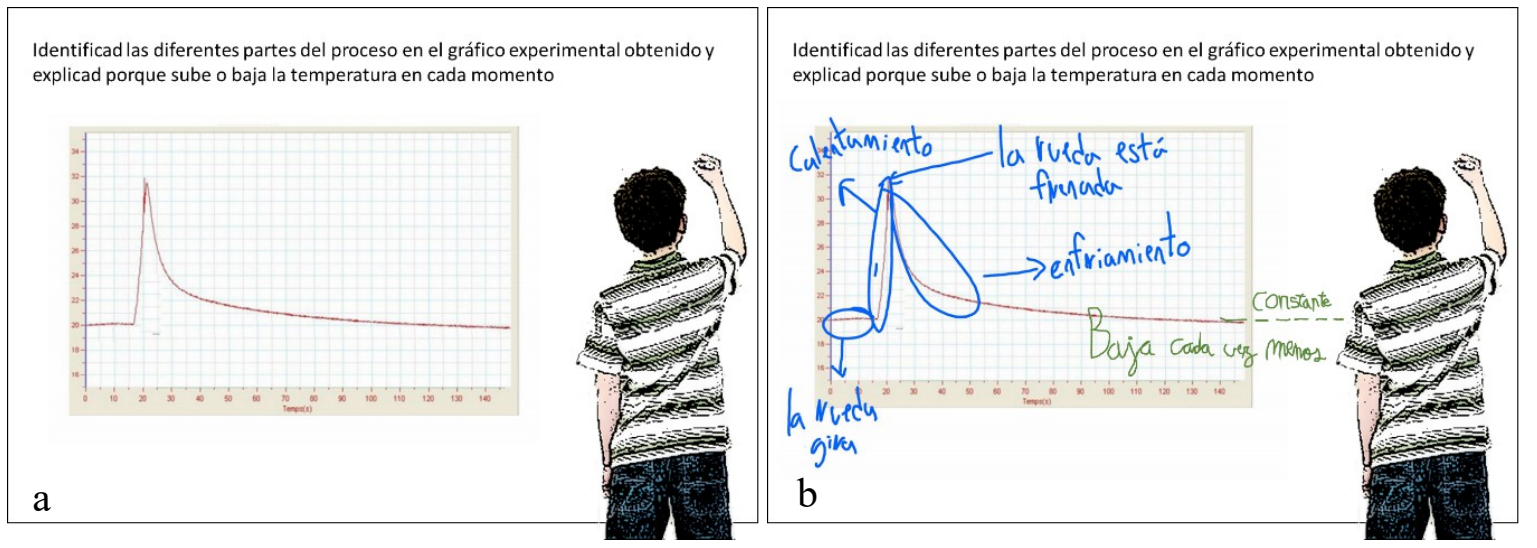

Figura 6. Izquierda: se proyecta el gráfico experimental obtenido de un experimento. Derecha: varios estudiantes señalan las diferentes partes del gráfico y las relacionan con las fases del fenómeno físico que están estudiando.

\section{Arrastrar objetos de la PDI para narrar y describir procesos, expresando su modelo (inicial o final)}

Finalmente, el último episodio seleccionado sucede cuando los estudiantes arrastran objetos que se encuentran en la pantalla, con el objetivo de narrar procesos dinámicos ya sea al inicio de una secuencia de enseñanza - aprendizaje para explorar las ideas previas del alumnado [1C] o al final, para construir un modelo de consenso [6C]. En la Figura 7 se muestra la captura de pantalla en dos momentos en los que se pide a un estudiante que arrastre la representación de unos átomos para explicar qué enlaces se rompen y qué enlaces se crean en una reacción química observada. 

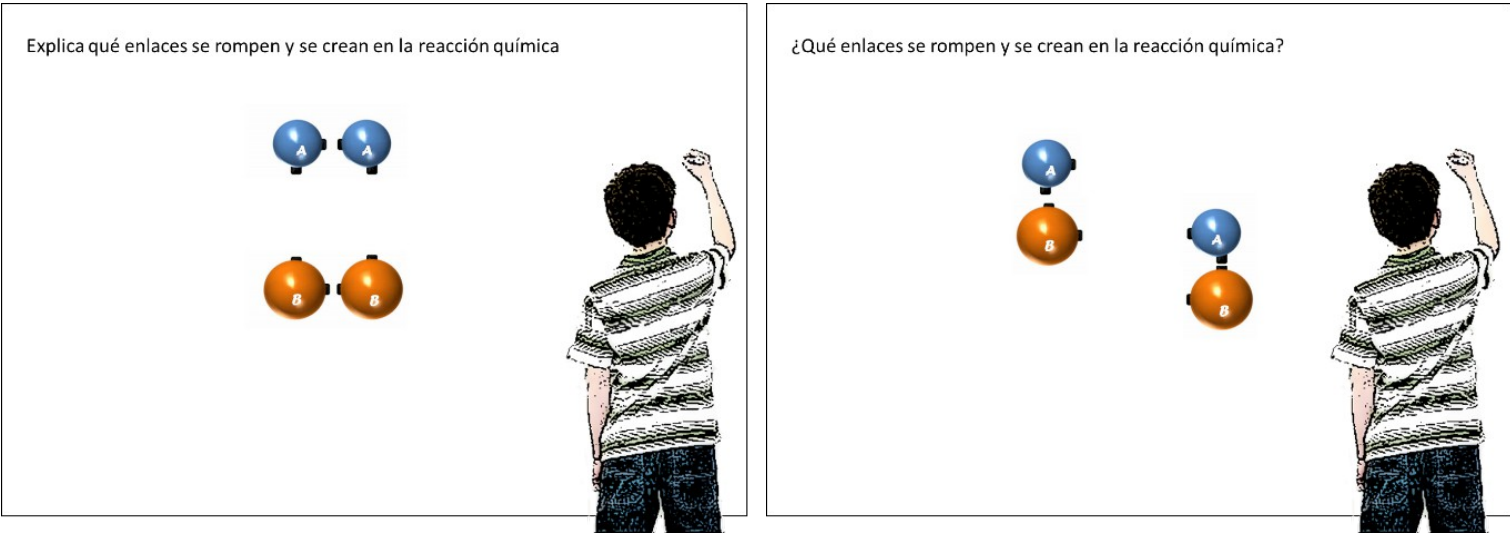

Figura 7. Izquierda: representación de los átomos y sus enlaces antes de una reacción química. Derecha: un estudiante ha arrastrado los átomos para explicar a los demás qué enlaces se rompen y qué enlaces se crean.

\section{Discusión de los episodios y conclusiones}

El análisis del uso de la PDI en el laboratorio digitalizado REVIR nos ha llevado a seleccionar cinco episodios especialmente seleccionados para entender cómo ayuda este dispositivo a la hora de promover prácticas de indagación y modelización en el aula de ciencias.

Uno de los denominadores comunes de estos cinco episodios es la naturaleza multimodal y multinivel de las representaciones (Lemke 1990; Johnstone 1991). En los casos en que el estudiante arrastra objetos en la pizarra (ya sea palabras escritas por el mismo o representaciones insertadas por el profesor), el propio hecho de arrastrar (acción [C]) se convierte en un proceso de comunicación científica en sí mismo: Así, a veces el estudiante arrastra para realizar representaciones narrativas (para "explicar", por ejemplo, cómo cree que se mueven las partículas en una reacción química), y a veces para comunicar representaciones conceptuales (para explicar, por ejemplo, cómo cree que unas maneras de calentar el agua se parecen más a otras). Estas estructuras composicionales narrativas y conceptuales propuestas desde la semiótica (Kress y van Leeuwen 1996), son especialmente útiles en el proceso de modelización de los estudiantes (Márquez et al. 2006).

Otro denominador común de los distintos episodios seleccionados es cómo la PDI ayuda a generar un espacio social para la construcción participativa y compartida del conocimiento, que suele partir de las producciones de los estudiantes que salen a escribir o dibujar a la pizarra que corresponden a sus concepciones o ideas iniciales. Pero a diferencia de lo que ocurre en la pizarra tradicional, los trazos introducidos a mano en la PDI (ya sean dibujos o palabras) se transforman en objetos que pueden ser movidos en la pantalla arrastrándolos (acción [C]). Este hecho, que podría parecer trivial, hace que ordenar estos objetos según un determinado criterio confiere un nuevo significado a la información final que se muestra, ofreciendo una oportunidad única para poder fomentar el diálogo sobre a las concepciones iniciales del alumnado. Son comunes las intervenciones de estudiantes tipo "yo arrastraría la palabra "agitar" más a la derecha, ya que creo que "agitar" y "bacer fuerza" se parecen más", al referirse a representaciones como la de la Figura 3. Pero, además, al poder almacenar y recuperar más adelante estas producciones (acción [E]) todavía ayuda más a confrontar las ideas expresadas al inicio de una secuencia de aprendizaje con las expresadas más adelante (ya sea en el transcurso de la misma sesión o en las siguientes). Esto es de gran utilidad a la hora de promover la coconstrucción de modelos (expresar, evaluar y revisar los modelos), tal como proponen Clement (2008) o Khan (2007).

La relación que se establece en el aula entre los estudiantes y los datos experimentales (los resultados obtenidos de una indagación como las Figuras 5 y 6) también toma una especial 
relevancia gracias a la PDI. El simple hecho de proyectar (acción [A]) los datos obtenidos en un sensor periférico (en la Fig. 5 es un sensor de distancia, en la Fig. 6 de temperatura), permite hacer partícipe a toda la clase de los resultados obtenidos por uno de los grupos que ha estado realizando las medidas con estos sensores. Pero si esto se podría hacer con un simple proyector, sobrescribir (acción [D]) sobre estos gráficos experimentales para señalar sus partes o destacar elementos importantes es una acción clave para facilitar la interpretación de estos resultados experimentales por parte de los estudiantes. Esto permite, de nuevo, generar oportunidades para promover la participación del alumnado en la interpretación de los resultados (por ejemplo, haciendo que varios estudiantes vayan saliendo, uno a uno, a señalar una de las partes del gráfico), de manera que en la clase ya no solo se proyectan gráficos como los de la Fig. 6a, sino auténticas composiciones colaborativas como las de la Fig. 6b. Si a esto se le añade la posibilidad que ofrece la PDI para arrastrar y soltar objetos (acción [C]), la discusión colaborativa también puede realizarse respecto al montaje experimental que se va a hacer (ver Fig. 4). Así, ofrecer que varios estudiantes salgan a la pizarra a arrastrar los objetos que representan los elementos del montaje experimental que se va a usar también puede ayudar, por ejemplo, a evidenciar que no siempre existe un único «método científico», sino que diversos montajes experimentales permiten responder a una misma pregunta investigable.

Finalmente, otro elemento común de los 5 episodios seleccionados y de las representaciones visuales «producto » de la interacción en el aula que se dan en cada uno de ellos (Figuras 3, 4, 5,6 y 7) es que en todas ellas se rompe el binomio tradicional que creaba el sistema proyectorpizarra tradicional entre la información externa y «verdadera» (representada por la información proyectada en el proyector) y la información interna y «en práctica» (que se encontraba en la pizarra tradicional y se generaba en el aula). Así pues, la PDI unifica estos dos espacios, ya que sobre la misma superficie se puede proyectar, manipular y generar nueva información, incrementando el valor y la autenticidad del conocimiento que se produce en el aula en relación con el conocimiento externo. Esta característica es esencial a la hora de ofrecer una visión de la práctica científica acorde con la construcción del conocimiento científico a nivel profesional según Evagorou, Erduran y Mäntylä (2015) y Kelly (2013).

En definitiva, las diversas acciones que se pueden realizar con la PDI permiten usar este dispositivo como herramienta mediadora de las interacciones que ocurren en el aula, que puede promover la construcción de un conocimiento científico en comunidad (coconstrucción del conocimiento). Así, los episodios seleccionados, más allá del ejemplo concreto del uso de la PDI para el desarrollo de un modelo científico particular, muestran cómo esta herramienta puede facilitar el desarrollo de investigaciones en el aula y la interpretación de datos provenientes de fenómenos naturales (indagación), y que el alumnado pueda hacerlo explicitando sus ideas científicas, refinarlas progresivamente a través de la interacción con el profesorado y con sus iguales (modelización).

Ciertamente, estos episodios seleccionados han sido identificados en un contexto educativo particular y, aunque puedan ser un ejemplo ilustrativo de cómo usar la PDI para promover la indagación y la modelización en el aula, no significa que sean la única manera en la que este dispositivo puede ser usado. Para ello, habrá que seguir estudiando cómo la PDI puede devenir una buena herramienta en una mayor diversidad de situaciones.

\section{Implicaciones y recomendaciones para el aula de ciencias}

Instalar una PDI en un aula no conlleva per se enseñar mejor ni que los estudiantes aprendan más ni mejor, más allá de los aspectos motivacionales que escapan del alcance de este trabajo. No obstante, evidenciar cómo la PDI puede contribuir a la mejora educativa no siempre es tarea fácil y por este los cinco episodios aquí mostrados y discutidos pretenden aportar una 
mirada a la PDI como herramienta para promover la modelización y la indagación. A pesar de que cada ejemplo se ciñe a un contenido científico muy concreto (trabajo, calor y temperatura en las Figuras 3 y 6, termorregulación en la Figura 4, cinemática en la Figura 5 y enlace químico en la 7), la manera de promover la interacción entre estudiantes, profesorado, datos experimentales, ideas y modelos, etc., es equiparable a muchos otros contenidos científicos.

Por ejemplo, promover que los estudiantes salgan a sobrescribir sobre información proyectada no es algo que pueda hacerse solo sobre un gráfico experimental, sino también sobre mapas, fotografías recogidas con microscopio, fotogramas de un vídeo, etc. Del mismo modo, que los estudiantes salgan a arrastrar ideas (representadas con imágenes o palabras) y agruparlas según su significado no es algo que solo sirva para clasificar mecanismos de transferencia de energía, sino que es una práctica genuinamente científica aplicable a múltiples contenidos. Igual como lo es la idea de elaborar predicciones para después compararlas con el resultado obtenido en un experimento, que gracias a la PDI permite capturar automáticamente todo lo que se ha escrito en la pizarra y recuperarlo más adelante.

Para aprovechar todo este potencial de la herramienta, es necesario que el profesorado se plantee cuándo y cómo se debe usar la PDI: qué ámbito de la práctica científica escolar se pretende desarrollar con la PDI (modelizar, indagar...); en qué momento de la secuencia de enseñanza y aprendizaje se pretende usar la PDI (exploraciones de ideas previas, estructuración de contenido...); qué representaciones visuales pueden servir mejor a los dos propósitos anteriores (estructuras composicionales narrativas o conceptuales); y qué acciones con la PDI permiten generar las representaciones visuales deseadas (arrastrar objetos, sobrescribir encima de una imagen...). Será necesario añadir a esta reflexión, aunque no haya sido objeto de estudio en el presente artículo, qué modelo o ideas científicas se pretende construir en el aula (relacionadas con una determinada disciplina), así como las principales dificultades que ofrecen al alumnado.

En definitiva, usar la PDI para promover la práctica científica en el aula implica un cierto manejo fluido de esta herramienta que es diferente según el modelo y se sofistica con el paso de los años (cada vez con más opciones de selección y trazo más completas, superficies multitáctiles e integración de dispositivos móviles, etc.). No obstante, situar la formación solo a este nivel técnico lleva a que no se aproveche las oportunidades que ofrece la PDI, de acuerdo con Hennessy y London (2013). Para aprovechar todo su potencial educativo es necesario promover espacios de diálogo en el que el profesorado pueda reflexionar acerca del cuándo y cómo usar la PDI en el aula de ciencias.

\section{Apoyos y agradecimientos}

Investigación financiada por el Ministerio de Economía y Competitividad (con referencia EDU2015-66643-C2-1-P) y realizada en el grupo consolidado ACELEC (2017SGR1399).

\section{Referencias}

Aflalo, E., Zana, L. \& Huri, T. 2017. The interactive whiteboard in primary school science and interaction. Interactive Learning Environments, 0(0), pp.1-14.

Area-Moreira, M., Hernández-Rivero, V. \& Sosa-Alonso, J.-J. 2016. Integración de las TIC en el aula. Comunicar, 47(24), pp.79-87.

Beauchamp, G. \& Parkinson, J. 2005. Beyond the "wow" factor: developing interactivity with the interactive whiteboard. School Science Review, 86(316), pp.97-104.

Bozzo, G., Grimalt-Álvaro, C. \& López, V. 2015. The uses of Interactive Whiteboard in a 
science laboratory. In C. Fazio \& R. M. Sperandeo Mineo, eds. GIREP-MPTL 2014 Proceedings. Palermo, pp. 549-558.

Chamizo, J.A. \& Izquierdo, M. 2005. Ciencia en contexto: una reflexión desde la filosofía. Alambique, 46(1), pp.9-17.

Clement, J.J. 2008. Student/Teacher Co-construction of Visualizable Models in Large Group Discussion. In J. J. Clement \& A. Rea-Ramirez, eds. Model Based Learning and Instruction in Science. Springer, pp. 11-22.

Couso, D. \& Garrido, A. 2016. Models and modelling in elementary school pre-service teacher education: the influence of teaching scenarios. In 11th ESERA Conference Selected Contributions. pp. 1-18.

Crook, S.J., Sharma, M.D. \& Wilson, R. 2014. An Evaluation of the Impact of 1:1 Laptops on Student Attainment in Senior High School Sciences. Dx.Doi.Org, 37(2), pp.272-293.

Duschl, R.A. \& Grandy, R.E. 2012. Two Views About Explicitly Teaching Nature of Science. Science \& Education, 22, pp.2109-2139.

Evagorou, M., Erduran, S. \& Mäntylä, T. 2015. The role of visual representations in scientific practices: from conceptual understanding and knowledge generation to "seeing" how science works. International Journal of STEM Education, 2(1), p.11.

Grimalt-Álvaro, C. 2016. La tecnologia a les classes de ciències de secundària: anàlisi dels processos de canvi en el professorat. Universitat Autònoma de Barcelona.

Gutiérrez, R. 2004. La modelización y los procesos de enseñanza / aprendizaje. Alambique Didàctica de las Ciencias Experimentales, 42, pp.8-18.

Hennessy, S. \& London, L. 2013. Learning from International Experiences with Interactive Whiteboards: The role of professional development in integrating the technology. OECD Education Working Papers, (89), p.33.

Higgins, S., Wall, K. \& Smith, H.J. 2005. "The visual helps me understand the complicated things": pupil views of teaching and learning with interactive whiteboards. British Journal of Educational Technology, 36(5), pp.851-867.

Izquierdo, M. et al. 1999. Caracterización y fundamentación de la ciencia escolar. Enseñanza de las ciencias, número ext (December 2015), pp.79-91.

Johnstone, A.H. 1991. Why is science difficult to learn? Things are seldom what they seem. Journal of Computer Assisted Learning, 7(2), pp.75-83.

Justi, R. 2006. La enseñanza de ciencias basada en la elaboración de modelos. Enseñanza de las ciencias, 24(2), pp.173-184.

Kelly, J. 2013. Inquiry teaching and learning: philosophical considerations. In M. R. Matthews, ed. Handbook of Historical and Philosophical Studies in Science Education. Pensylvania: Springer.

Kennewell, S. \& Beauchamp, G. 2007. The features of interactive whiteboards and their influence on learning. Learning, Media and Technology, 32(3), pp.227-241.

Khan, S. 2007. Model-Based Inquiries in Chemistry. Science Education, 91(1), pp.877-905.

Koehler, M.J. \& Mishra, P. 2009. What is Technological Pedagogical Content Knowledge (TPACK)? J. M. Spector et al., eds. Contemporary Issues in Technology and Teacher Education, 9(1), pp.60-70.

Kress, G. \& van Leeuwen, T. 1996. Reading Images. The Grammar of Visual Design, New York: 
Routledge.

Kung Teck, W. 2013. Affordances of interactive whiteboards and associated pedagogical practices: perspectives of teachers of Science with children aged five to six years. The Turkish Online Journal of Educational Technology, 12(1), pp.1-8.

Lemke, J.L. 1990. Talking science: Language, learning, and values,

Linn, M. 2003. Technology and science education: starting points, research programs, and trends. International Journal of Science Education, 25(6), pp.727-758.

López Simó, V. et al. 2017. El papel de las TIC en la enseñanza de las ciencias en secundaria desde la perspectiva de la práctica científica. In X Congreso Enseñanza de las Ciencias. p. 49.

Márquez, C., Izquierdo, M. \& Espinet, M. 2006. Multimodal science teachers' discourse in modeling the water cycle. Science Education, 90(2), pp.202-226.

Mercer, N., Hennessy, S. \& Warwick, P. 2010. Using interactive whiteboards to orchestrate classroom dialogue. Technology, Pedagogy and Education, 19(2), pp.195-209.

Miller, D. et al. 2005. How can the use of an interactive whiteboard enhance the nature of teaching and learning in secondary mathematics and modern foreign languages $\square$ ?,

Minner, D.D., Levy, A.J. \& Century, J. 2010. Inquiry-based science instruction-what is it and does it matter? Results from a research synthesis years 1984 to 2002. Journal of Research in Science Teaching, 47(4), pp.474-496.

Mortimer, E.F. \& Scott, P.H. 2003. Meaning Making in Secondary Science Classrooms, McGraw-Hill International.

Murcia, K. 2014. Interactive and multimodal pedagogy: A case study of how teachers and students use interactive whiteboard technology in primary science. Australian Journal of Education, 58(1), pp.74-88.

National Research Council 2012. A Framework for K-12 Science Education: Practices, Crosscutting Concepts and Core Ideas Committee on a Conceptual Framework for New K-12 Science Education Standards. Board on Science Education. Division of Behavioral and Social Sciences and Education., ed., Washington, DC: The National Academies Press.

Ormanci, U. et al. 2015. A Thematic Review of Interactive Whiteboard Use in Science Education: Rationales, Purposes, Methods and General Knowledge. Journal of Science Education and Technology, 24(5), pp.532-548.

Osborne, J. 2014. Teaching Scientific Practices: Meeting the Challenge of Change. Journal of Science Teacher Education, 25, pp.177-196.

Osborne, J. \& Hennessy, S. 2003. Literature Review in Science Education and the Role of ICT: Promise, Problems and Future Directions.,

Pedró, F. 2011. Tecnología y escuela: lo que funciona y por qué, Fundación Santillana.

Pintó, R., Couso, D. \& Hernández, M.I. 2010. An inquiry-oriented approach for making the best use of ICT in the classroom. eLearning Papers, 20(July), pp.1-14.

Romero, M. \& Quesada, A. 2014. Nuevas tecnologías y aprendizaje significativo de las ciencias. Enseñanza de las ciencias: Revista de investigación y experiencias didácticas, 32(1), pp.101115.

Roschelle, J.M. et al. 2001. Changing how and what children learn in school with computerbased technologies. The Future of children, 10(2), pp.76-101. 
Smetana, L.K. \& Bell, R.L. 2011. Computer Simulations to Support Science Instruction and Learning: A critical review of the literature. International Journal of Science Education, 34(9), pp.1337-1370.

Vygotsky, L.S. 1978. Mind in society: The development of higher psychological processes.

Webb, M.E. 2005. Affordances of ICT in science learning: implications for an integrated pedagogy. International Journal of Science Education, 27(6), pp.705-735.

Windschitl, M., Thompson, J. \& Braaten, M. 2008. Beyond the Scientific Method: ModelBased Inquiry as a New Paradigm of Preference for School Science Investigations. Science Education, 92(5), pp.941-967. 